\title{
Zinc Promotes Osteoblast Differentiation in Human Mesenchymal Stem Cells Via Activation of the cAMP-PKA-CREB Signaling Pathway
}

\author{
Kwang Hwan Park, ${ }^{1, *}$ Yoorim Choi, ${ }^{1,2,{ }^{*}}$ Dong Suk Yoon, ${ }^{3}$ Kyoung-Mi Lee, ${ }^{1,4}$ \\ Dohyun Kim, and Jin Woo Lee ${ }^{1,2,4}$
}

The crucial trace element zinc stimulates osteogenesis in vitro and in vivo. However, the pathways mediating these effects remain poorly understood. This study aimed to investigate the effects of zinc on osteoblast differentiation in human bone marrow-derived mesenchymal stem cells (hBMSCs) and to identify the molecular mechanisms of these effects. In hBMSCs, zinc exposure resulted in a dose-dependent increase in osteogenesis and increased mRNA and protein levels of the master transcriptional factor RUNX2. Analyzing the upstream signaling pathways of RUNX2, we found that protein kinase A (PKA) signaling inhibition blocked zinc-induced osteogenic effects. Zinc exposure increased transcriptional activity and protein levels of phospho-CREB and enhanced translocation of phospho-CREB into the nucleus. These effects were reversed by H-89, a potent inhibitor of PKA. Moreover, zinc exposure led to dose-dependent increases in levels of intracellular cyclic adenosine monophosphate (cAMP). These findings indicate that zinc activates the PKA signaling pathway by triggering an increase in intracellular cAMP, leading to enhanced osteogenic differentiation in hBMSCs. Our results suggest that zinc exerts osteogenic effects in hBMSCs by activation of RUNX2 via the cAMP-PKACREB signaling pathway. Zinc supplementation may offer a promise as a potential pharmaceutical therapy for osteoporosis and other bone loss conditions.

Keywords: zinc, bone formation, osteoblast, RUNX2, protein kinase A

\section{Introduction}

$\mathrm{O}$ STEOPOROSIS OCCURS AS a result of an imbalance between osteoblast and osteoclast activity [1-3]. In order to maintain bone homeostasis, bone loss due to osteoclast activity must be compensated for by bone formation by osteoblasts. During osteogenesis, a number of genes are upregulated, including COLIA1, ALPL, OPN, BGLAP, IBSP, and $O N$ [4]. In this investigation, in order to identify novel genes involved in osteogenesis, we first performed an analysis using two sets of microarray data from Gene Expression Omnibus Data Sets; these were comparative microarrays for human mesenchymal stromal cells cultured with basal medium or osteogenic medium (OM) [5,6]. Differentially expressed genes (DEGs) were identified through statistical analyses performed using R software. Through these analyses, we identified intersections between the two DEG sets. Among these, a topranked upregulated gene during osteoblast differentiation was the metallothionein gene (MT), which encodes a zinc-binding protein responsible for regulating intracellular zinc. Consistent with this finding, a previous study using a mouse model reported $M T$ knockout to be associated with low plasma zinc concentration and impaired skeletal growth [7].

Although zinc is a trace element, it is a constituent of numerous enzymes and proteins that perform multiple biological functions necessary for maintaining health. With respect to skeletal biology, zinc plays crucial roles in bone metabolism [8] and normal growth processes [9]. Several studies have reported that dietary deficiency of zinc disrupts normal bone growth and maturation in humans, as well as in animal models $[10,11]$. In addition, zinc has been found to increase alkaline phosphatase (ALP) activity [12] and collagen synthesis during bone formation [13]. In clinical studies, zinc intake has been found to positively correlate with bone mineral density in premenopausal women [14] and to negatively correlate with bone loss in postmenopausal women [15]. Taken together,

\footnotetext{
${ }^{1}$ Department of Orthopaedic Surgery, Yonsei University College of Medicine, Seoul, South Korea.

${ }^{2}$ Brain Korea 21 Plus Project for Medical Sciences, Yonsei University College of Medicine, Seoul, South Korea.

${ }^{3}$ Department of Internal Medicine, Brody School of Medicine at East Carolina University, Greenville, North Carolina.

${ }^{4}$ Severance Biomedical Science Institute, Yonsei University College of Medicine, South Korea.

*These authors contributed equally to this work.
} 
these findings suggest that zinc likely plays an important role in the signaling pathways of osteogenesis.

In another recent study, the zinc transporter protein ZIP13 was shown to be involved in the BMP/TGF- $\beta$ signaling pathway during connective tissue development, a process which includes osteoblast maturation [16]. Another zinc transporter, ZIP14, has been proposed to control G-proteincoupled receptor-mediated signaling, which is required for mouse systemic growth [17]. Although previous studies have reported the existence of a relationship between zinc and osteogenesis, the molecular mechanisms accounting for this relationship have remained to be elucidated.

In this study, we aimed to characterize the effects of zinc exposure on osteoblast differentiation in human bone marrow-derived mesenchymal stem cells (hBMSCs). We focused on investigating the mechanistic role of zinc with respect to RUNX2, a master transcriptional factor in osteogenesis. Our hypothesis was that zinc exposure might increase RUNX2 levels via the cAMP-PKA-CREB signaling pathway, thereby inducing enhanced osteoblast differentiation and stimulating osteogenesis.

\section{Materials and Methods}

\section{Cell culture and reagents}

Human bone marrow aspirates from the posterior iliac crest were obtained from seven adult donors (four males and three females; age range: $50-60$ years old). From these bone marrow samples, hBMSCs were isolated based on cell ability to adhere to a plastic culture flask. Following selection, cells were maintained in low-glucose Dulbecco's modified Eagle medium (DMEM-LG) (Gibco; Grand Island, NY) supplemented with $10 \%$ fetal bovine serum (FBS) (Gibco) and $1 \%$ antibiotic-antimycotic solution (Carlsbad, $\mathrm{CA})$ at $37^{\circ} \mathrm{C}$ in a $5 \% \mathrm{CO}_{2}$ atmosphere. hBMSCs were grown to $70 \%-80 \%$ confluency and then harvested by incubation with $0.05 \%$ trypsin/EDTA (Invitrogen, Carlsbad, CA) followed by centrifugation at 1,300 revolutions per minute (rpm) for $3 \mathrm{~min}$. Cells obtained through this process were then replated at a density of 5,000 cells $/ \mathrm{cm}^{2}$ and subcultured when they were $80 \%-90 \%$ confluent. hBMSC characteristics were confirmed for this protocol in our previous studies [18-20]. To determine the optimal concentration of $\mathrm{ZnSO}_{4}$ (Sigma; St. Louis, MO) for enhancing osteogenic differentiation in hBMSCs in vitro, cultured cells were assigned to one of the following study groups: control group or one of five experimental groups $\left(\mathrm{ZnSO}_{4}\right.$ treatment groups: 10, 20, 50, 100, and $200 \mu \mathrm{M}$ ). AKT1/2 kinase inhibitor (A6730; Sigma), H-89 (Sigma), was used at a concentration of $0.1 \mu \mathrm{M}$, and DKK1 (R\&D Systems, Minneapolis, MN) was used at a concentration of $0.1 \mu \mathrm{g} / \mathrm{mL}$ in hBMSCs. This study protocol was approved by the Institutional Review Board (IRB) of the Yonsei University College of Medicine (IRB No. 4-2017-0232) and informed consent was obtained from all patients. All methods were performed according to the relevant guidelines and regulations of the institution.

\section{In vitro osteogenesis and adipogenesis}

The medium used for stimulating in vitro osteogenesis by hBMSCs has been described previously [21]. In brief, hBMSCs were seeded at $4 \times 10^{4}$ cells/well in 24 -well plates or
$1 \times 10^{4}$ cells/well in 96-well plates. To induce osteogenic differentiation, hBMSCs were maintained for 5-10 days in OM [DMEM-LG containing 10\% FBS, $1 \%$ antibiotic-antimycotic solution, $10 \mathrm{mM} \beta$-glycerophosphate (Sigma), $100 \mathrm{nM}$ dexamethasone (Sigma), and $50 \mu \mathrm{g} / \mathrm{mL}$ ascorbic acid (Gibco)]. To adipogenic differentiation, hBMSCs were maintained for 721 days in adipogenic medium (AM) (DMEM-LG containing $10 \%$ FBS, $1 \%$ antibiotic-antimycotic solution, $0.5 \mathrm{mM}$ isobutylmethylxanthine [Sigma], $1 \mu \mathrm{M}$ dexamethasone [Sigma], $200 \mu \mathrm{M}$ indomethacin [Sigma], and $5 \mu \mathrm{M}$ insulin [Sigma]). For each experimental group, $\mathrm{ZnSO}_{4}$-supplemented medium was replaced every 2 days during this differentiation period.

\section{Cell viability assay}

Cell viability was evaluated using an EZ-Cytox Cell Viability Assay Kit (Daeil Lab Service, Seoul, Korea) according to the manufacturer's instructions. For this assay, hBMSCs were seeded in 12-well culture plates at a density of $1 \times 10^{4}$ cells/well. Cells were then maintained in DMEMLG for 5 days, and medium was replaced once per day during the assay period. As detailed in the kit instructions, at the end of the assay period, after cells were washed with phosphate-buffered saline (PBS), $10 \mu \mathrm{L}$ of EZ-Cytox (tetrazolium salts) solution was added to each well, and these were incubated at $37^{\circ} \mathrm{C}$ for $3 \mathrm{~h}(\mathrm{~h})$. After incubation, conditioned media were transferred to 96-well plates. Absorbance was then measured at $410 \mathrm{~nm}$. All samples were tested in triplicate.

\section{Colony-forming units-fibroblast assay}

Human bone marrow mononuclear cells (hBMMs) were seeded at $5 \times 10^{5}$ in 100 -mm culture dishes and maintained in DMEM-LG supplemented with $20 \%$ FBS for 12 days. $\mathrm{ZnSO}_{4}$-treated media was replaced every 2 days during fibroblast colony formation for $\mathrm{ZnSO}_{4}$-treated $\mathrm{hBMMs}$. Subsequently, cells were fixed in a 1:1 acetone: methanol fixative, stained with $20 \%$ crystal violet solution (Merck; Darmstadt, Germany) for $30 \mathrm{~min}$ in darkness, and washed in distilled water (DW). Human bone marrow fibroblast colony-forming ability of the stained cells was then evaluated and counted in triplicate.

\section{Flow cytometry}

hBMSCs were cultured with $100 \mu \mathrm{M} \mathrm{ZnSO}_{4}$ for 3 days and harvested with $0.02 \%$ EDTA and washed twice in fluorescence-activated cell sorting (FACS) buffer (PBS containing $1 \%$ FBS and $0.05 \%$ sodium azide). The hBMSCs $\left(2 \times 10^{5}\right)$ were stained with CD73-PE (Miltenyi Biotec, Auburn CA), PE-mouse-IgG2a isotype control (Miltenyi Biotec), CD90-FITC (Miltenyi Biotec), FITC-mouse IgG2a isotype control (Miltenyi Biotec), CD105-APC (Miltenyi Biotec), CD146-APC (Biolegend, San Diego, CA), and APC-mouse IgG1 isotype control (Miltenyi Biotec) for $1 \mathrm{~h}$ at $4{ }^{\circ} \mathrm{C}$. Thereafter, the stained cells were washed with PBS, centrifuged at $300 \mathrm{~g}$ for $5 \mathrm{~min}$, and then resuspended in $500 \mu \mathrm{L}$ of ice-cold FACS buffer. The samples were subjected to FACS analysis using a FACS Verse ${ }^{\mathrm{TM}}$ flow cytometer (BD Biosciences). With an unstained sample $\left(3 \times 10^{5}\right)$ as the negative control, the stained cells were analyzed using FlowJo software (FlowJo LLC, Ashland, OR). 


\section{ALP/Alizarin red S staining and quantification}

For ALP staining, after fixation in a 2:3 citrate buffer: acetone fixative, hBMSCs treated with test concentrations of $\mathrm{ZnSO}_{4}$ were stained for ALP using an alkaline staining solution mixed with fast blue RR salt (Sigma) in naphthol AS-MX phosphate alkaline solution (Sigma) for $30 \mathrm{~min}$ in darkness. After washing in DW, cells were stained with Mayer's hematoxylin solution (Sigma) for $5 \mathrm{~min}$ and then rinsed in tap water. ALP activity was normalized to Alamar blue. For alizarin red S staining, after cells were fixed in icecold $70 \%$ ethanol, freshly prepared $3 \%$ alizarin red S solution (weight/volume [wt/vol]) (Sigma) was added. Then samples were incubated for $30 \mathrm{~min}$. For quantification of alizarin red S, absorbance was detected at $595 \mathrm{~nm}$, following destaining with $10 \%$ cetylpyridinium chloride monohydrate (Sigma) for 10-20 min.

\section{Oil red O staining for adipogenic differentiation}

hBMSCs were rinsed with PBS, fixed in $10 \%$ neutralbuffered formalin buffer (Sigma) for 30 min, washed by DW. Then, cells were stained with and $0.18 \%$ oil red O solution (sigma) for $1 \mathrm{~h}$, followed by repeated washing with DW. For quantitative analysis of stained cells, absorbance was detected at $500 \mathrm{~nm}$ after destaining with $100 \%$ isopropanol for $30 \mathrm{~min}$.

\section{Quantitative real-time polymerase chain reaction}

Quantitative real-time polymerase chain reaction (qPCR) analysis was performed as previously described [19]. In brief, total RNA was isolated using a RNeasy kit (Qiagen; Valencia, $\mathrm{CA}$ ) according to the manufacturer's instructions. One microgram of total RNA was reverse-transcribed using an Omniscript Kit (Qiagen). The primer sets (Bioneer; Seoul, Korea) were as follows: GAPDH (P267613), PPAR- $\gamma$ (P102359), RUNX2 (P229954), ALPL (P324388), COL1A1 (P157768), Osteonectin (P279949), Bone sialoprotein (P188040), and PKA (P312739). There were no validated primers for Osteopontin and Osteocalcin. Thus, the primers for these were designed as follows: Osteopontin, 5'-CCGTTGCCCAGGAC CTGAA-3' (sense) and 5'-TG TGGCTGTGGGTTTCAGCA$3^{\prime}$ (antisense); Osteocalcin, 5'-AGAGCCCCAGTCCCCTA CC C-3' (sense) and 5'-AGGCCTCCTGAAAGCCGATG-3' (antisense). Mean cycle threshold values from triplicate $(n=3)$ measurements were used to calculate gene expression, with normalization to GAPDH as an internal control.

\section{Western blot}

For total protein extraction, hBMSCs were lysed in passive lysis buffer (Promega; Madison, WI). Protein concentrations were determined with a Bio-Rad protein assay (Bio-Rad Laboratories, Inc.; Hercules, CA), and 10-30 $\mu \mathrm{g}$ of protein was analyzed by $10 \%$ sodium-dodecyl sulfate-polyacrylamide gel electrophoresis (Sigma). Transferred membranes were blocked with 5\% skim milk (BD; Sparks, MD) or 5\% bovine serum albumin (BSA) (Sigma), and incubated for $12 \mathrm{~h}$ with antibodies against RUNX2 (Millipore; San Diego, CA), PKA (Santa Cruz Biotechnology; Santa Cruz, CA), p-CREB (Cell Signaling; Danvers, MA), CREB (Cell Signaling), active $\beta$-CATENIN (Cell Signaling), $\beta$-CATENIN (Abcam; Cambridge, United
Kingdom), p-ERK1/2 (Abcam), ERK1/2 (Abcam), pSMAD2 (Cell Signaling), SMAD2 (Abcam), p-SMAD 1/5/8 (Abcam), SMAD 1/5/8 (Abcam), p-AKT (Cell Signaling), AKT (Cell Signaling), p-GSK-3 $\beta$ (Santa Cruz Biotechnology), GSK-3 $\beta$ (Santa Cruz Biotechnology), PPAR- $\gamma$ (Santa Cruz Biotechnology), FABP4 (Santa Cruz Biotechnology), Lamin B (Santa Cruz Biotechnology), and lactate dehydrogenase (Santa Cruz Biotechnology). Membranes were further probed with an antibody against HSP90 (Santa Cruz Biotechnology) and $\beta$-ACTIN (Santa Cruz Biotechnology), which served as a loading control.

\section{Nuclear and cytosolic fractionation}

hBMSCs were collected by trypsinization and washed with PBS three times before nuclear and cytosolic fractionation. Nuclear and cytoplasmic fractionation was performed using the NE-PER Nuclear and Cytoplasmic Extraction Reagents kit (Thermo Fisher Scientific; Rockford, IL) according to the manufacturer's instructions. Each separated protein was analyzed by western blot analysis.

\section{Lentiviral small hairpin RNA transduction}

Methods for lentiviral small hairpin RNA (shRNA) transduction and infection were previously described [22]. In brief, $3 \times 10^{6}$ HEK-293FT cells per $100-\mathrm{mm}$ dish were transfected with lentiviral shPKA vectors (TRCN0000356094 or TRCN0000233527, Sigma) using Lipofectamine 2000 (Invitrogen). After 6 h, HEK-293FT cells were sustained in DMEM-LG supplemented with 10\% FBS for 2 days, and then the supernatant fractions were collected. For lentivirus infection, $5 \times 10^{4}$ cells per well were seeded on six-well plates and then exposed to virus-containing supernatants. Lentivirus-infected cells were selected using puromycin $(10 \mathrm{mg} / \mathrm{mL})($ Sigma $) 72 \mathrm{~h}$ later.

\section{Immunocytochemistry}

hBMSCs were seeded at 1,000 cells $/ \mathrm{cm}^{2}$ onto four-well glass chamber slides (Nalge Nunc International; Rochester, $\mathrm{NY}$ ), and the cells were incubated in a $5 \% \mathrm{CO}_{2}$ incubator at $37^{\circ} \mathrm{C}$. After an overnight incubation, the cells were washed with PBS, followed by fixation with $4 \%$ paraformaldehyde (Sigma) for $30 \mathrm{~min}$. Permeabilization was performed with $0.25 \%$ Triton X-100 in PBS for $10 \mathrm{~min}$, followed by blocking for $1 \mathrm{~h}$ with 3\% BSA in PBS. The cells were incubated with 1:100 dilution of primary antibodies against CREB (Cell Signaling) overnight at $4^{\circ} \mathrm{C}$. After washing three times with PBS, the cells were incubated with fluorescein isothiocyanate (FITC)-conjugated secondary antibody in a 1:5,000 dilution in 3\% BSA-containing PBS for $1 \mathrm{~h}$ at room temperature in darkness. The nuclei were stained with 4,6-diamidino-phenyindole (Sigma) and then examined using a confocal microscope (LSM700, Carl Zeiss MicroImaging GHBH; Jena, Germany).

\section{Luciferase reporter assay}

hBMSCs were seeded at $1 \times 10^{5}$ cells per well in six-well plates, and cells were transfected with $1 \mathrm{mg}$ of either CREB (SwitchGear Genomics; Menlo Park, CA), AKT (SwitchGear Genomics), TOP-flash, or FOP-flash reporter vectors, 
with $1 \mathrm{ng}$ of pRL-SV40 as control using Lipofectamine 2000. After $24 \mathrm{~h}$, the cells were maintained for 3 days in OM with $\mathrm{ZnSO}_{4}$ or $\mathrm{H}-89$. The cells were analyzed using a simple dual-luciferase assay (Promega).

\section{Measurement of intracellular cyclic adenosine monophosphate}

Intracellular cyclic adenosine monophosphate (cAMP) was measured with the direct cAMP ELISA kit (Enzo Life Sciences; Plymouth Meeting, PA). In brief, cell culture supernatants were loaded into plates, treated with antibody, and incubated at room temperature for $2 \mathrm{~h}$ on a microplate shaker. Then, plates were read with a spectrophotometric microplate reader at $405 \mathrm{~nm}$, and cAMP levels were analyzed according to the manufacturer's protocol.

\section{Phosphodiesterase activity assay}

Phosphodiesterase (PDE) activity was measured by PDE activity assay kit (Colorimetric) (Abcam; ab139460). In brief, $1 \times 10^{6}$ cells were homogenized in DW and disrupted by sonication. The supernatant was desalted by gel filtration, and then PDE activity was measured according to manufacturer's protocols. IBMX $(10 \mu \mathrm{M})$, which is a PDE inhibitor, was treated as negative control.

\section{Statistical analyses}

Data are presented as mean \pm standard deviation. We performed the Shapiro-Wilk normality test for checking normal distributions of the groups. If normality tests passed, two-tailed, unpaired Student's $t$-test, and if normality tests failed, Mann-Whitney tests were used for the comparisons between two groups. For more than two groups, we used one-way ANOVA if normality tests passed, followed by Tukey's multiple comparison test for all pairs of groups. The GraphPad PRISM version 6.0 was used for data management and statistical analyses. Values of $* P<0.05$ or $* * P<0.01$ were considered statistically significant.

\section{Results}

\section{Zinc promotes osteoblast differentiation in hBMSCs}

To determine whether zinc is cytotoxic to hBMSCs, we examined the viability of the cells. hBMSCs treated with up to $100 \mu \mathrm{M} \mathrm{ZnSO}{ }_{4}$ were viable for 5 days (Fig. 1A). We then examined the fibroblast colony-forming abilities exposed to a range of $\mathrm{ZnSO}_{4}$ concentrations using $\mathrm{hBMMs}$ for 12 days. The results of these colony-forming unit-fibroblast assays revealed that $\mathrm{ZnSO}_{4}$ had no effect on the number of colony-forming cells, compared with cells not treated with $\mathrm{ZnSO}_{4}$ (Fig. 1B). Also, we analyzed the expression of MSC-associated markers using flow cytometry in the absence or presence of $\mathrm{ZnSO}_{4}$. As a result, $\mathrm{ZnSO}_{4}$ treatment did not change expression pattern of MSC-associated markers (Supplementary Fig. S1; Supplementary Data are available online at www.liebertpub.com/ scd). We then investigated the effects of zinc on osteoblast differentiation in hBMSCs in vitro by exposing cells to OM in the presence or absence of zinc. Zinc treatment was found to promote osteoblast differentiation in a dose-dependent manner, as shown by an increase in the amount of ALP (Fig. 1C).
Alizarin red S staining and activity were also increased by zinc in a dose-dependent manner (Fig. 1D). In addition, we have confirmed that zinc has no effect on lipid drop formation and expressions of adipogenic-associated proteins (Supplementary Fig. S2). These results suggest that zinc enhances osteoblast differentiation of hBMSCs.

\section{Zinc enhances expression of RUNX2 and phosphorylation of PKA during osteoblast differentiation in hBMSCs}

In the first set of experiments, we confirmed that $100 \mu \mathrm{M}$ $\mathrm{ZnSO}_{4}$ enhanced osteogenic differentiation, while $\mathrm{ZnSO}_{4}$ had no effects on proliferation and fibroblast colonyforming ability. Thus, we designated $100 \mu \mathrm{M} \mathrm{ZnSO}_{4}$ as the maximal concentration that was nontoxic to hBMSCs. To gain insight into the molecular mechanisms mediating the osteogenic effects of zinc, we then analyzed mRNA levels of the master transcriptional factor $R U N X 2$ during osteoblast differentiation in the presence of $\mathrm{ZnSO}_{4}$. During periods of osteogenesis in hBMSCs, mRNA levels of RUNX2 gradually increased until day 3 , followed by a decrease thereafter, and expression of $R U N X 2$ was higher in the presence of $\mathrm{ZnSO}_{4}$ than OM without supplemental zinc (Fig. 2A). As expected, the overall protein levels of RUNX2 were enhanced by treatment with $\mathrm{ZnSO}_{4}$ during osteogenesis (Fig. 2B). Among the many genes related to osteoblast differentiation, mRNA levels of RUNX2 and its target genes, such as $A L P, C O L 1 a 1, O P N, O N, B S P$, and $O C N$, were increased in the presence of zinc (Fig. 2C). It has been reported that RUNX2 expression is regulated by several signaling pathways such as BMP, WNT, TGF- $\beta$, PTHrP, IGF, and FGF [23-27]. To analyze how zinc enhances RUNX2 expression, we screened the downstream targets of each signaling pathway involved in osteogenic differentiation in hBMSCs. $\mathrm{ZnSO}_{4}$ was not found to impact downstream targets of the BMP, TGF- $\beta$, or FGF signaling pathways, while zinc exposure did increase phosphorylation of PKA and CREB, well-known to be downstream of the PTHrP pathway during osteogenic differentiation (Supplementary Fig. S3A and Fig. 2B). $\mathrm{ZnSO}_{4}$ also activated AKT and $\beta$-CATENIN, downstream targets of the IGF pathway and WNT pathway (Supplementary Fig. S3B). These results indicate that $\mathrm{ZnSO}_{4}$ promotes osteogenic differentiation via RUNX2 upregulation.

\section{PKA inhibition abolishes the osteogenic effect of zinc in hBMSCs}

To investigate which signaling pathway could regulate RUNX2 expression induced by $\mathrm{ZnSO}_{4}$, we targeted specific signaling pathways using inhibitors during treatment with varying doses of $\mathrm{ZnSO}_{4}$. First, we selected inhibitor concentrations for each signaling pathway to efficiently suppress osteogenic differentiation. Interestingly, zinc exposure did not reverse the inhibition of early and late osteogenic effects by H-89, an inhibitor of PKA (Fig. 3A, B). However, WNT- and AKT-inhibited osteogenic effects were reversed by zinc treatment in a dose-dependent manner (Supplementary Fig. S4A, B). Thus, we hypothesized that PKA signaling might be the main signaling pathway for zincinduced osteogenic differentiation in hBMSCs. To test this 
A
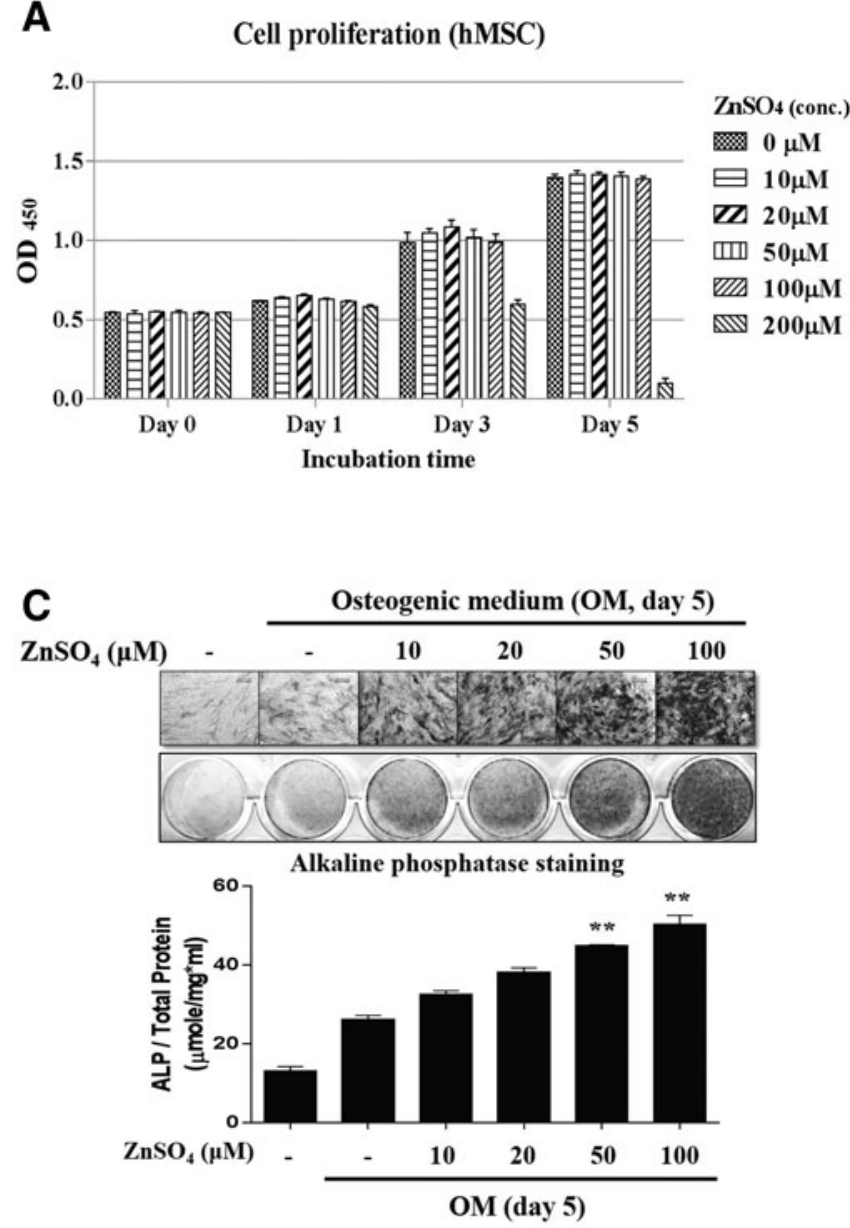

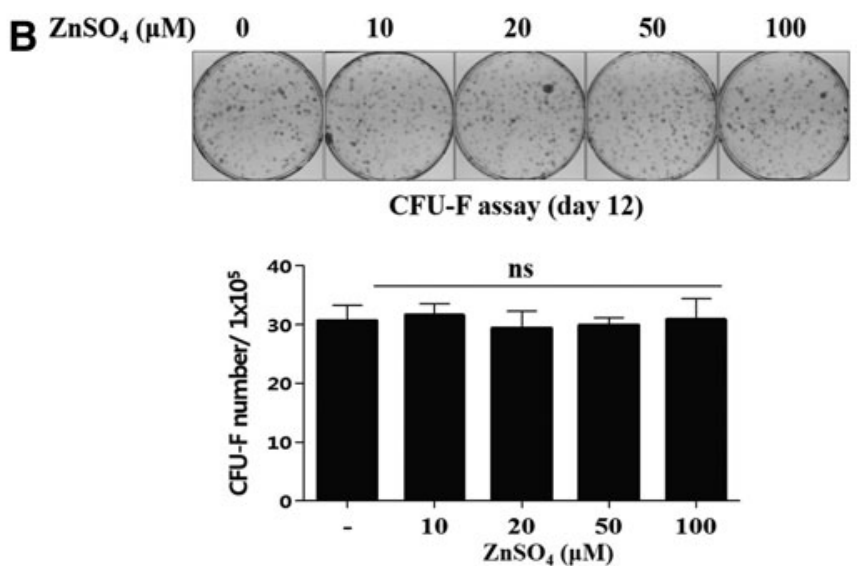

D $\mathrm{ZnSO}_{4}(\mu \mathrm{M})$
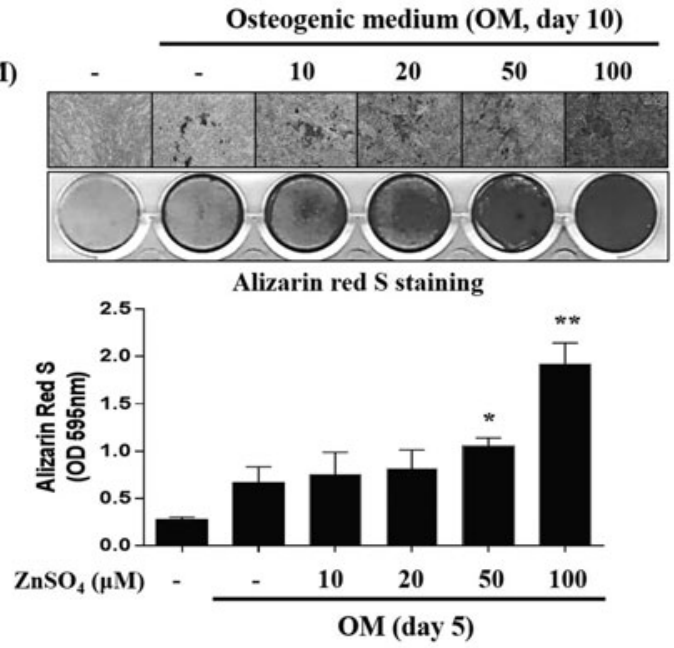

FIG. 1. The effects of zinc on proliferation, fibroblast colony-forming ability, and osteogenic differentiation. (A) hBMSCs were cultured with various concentrations of $\mathrm{ZnSO}_{4}$ for 5 days. Cell viability was measured using an EZ-Cytox cell viability kit. (B) Fibroblast-colony forming abilities were compared for $\mathrm{hBMMs}$ treated with various concentrations of $\mathrm{ZnSO}_{4}$ for 12 days using crystal violet staining (upper panel). The CFU-F numbers were counted by three observers (lower panel, $n=3$, in triplicate). (C) hBMSCs $\left(8 \times 10^{4}\right.$ cells/well in 12-well plates) were cultured for 5 days with OM and various concentrations of $\mathrm{ZnSO}_{4}$. ALP staining was performed (upper panel) and the amounts of ALP were measured to determine the extent of early osteoblast differentiation (lower panel). (D) hBMSCs $\left(8 \times 10^{4}\right.$ cells/well in 12-well plates) were cultured for 10 days with OM and various concentrations of $\mathrm{ZnSO}_{4}$. Alizarin red S staining was performed to detect mineral deposition (upper panel). For quantitative analysis of alizarin red S staining, absorbance was measured at $595 \mathrm{~nm}$, following destaining with $10 \%$ cetylpyridinium for 30 min (lower panel). Data are presented as the mean $\pm \mathrm{SD}$ of at least three independent experiments. $* P<0.05, * * P<0.01$ compared with control. hBMSC, human bone marrow-derived mesenchymal stem cells; OM, osteogenic medium; ALP, alkaline phosphatase; CFU-F, colony-forming units-fibroblast; hBMMs, human bone marrow mononuclear cells; SD, standard deviation.

hypothesis, we infected hBMSCs with shRNA targeting PKA. The efficiency of the shPKA was then confirmed by qPCR and western blot analysis (Fig. 3C). Then, we treated PKA-knockdown hBMSCs with $\mathrm{ZnSO}_{4}$ during osteogenic differentiation for 5-10 days. During osteogenic differentiation of hBMSCs in the presence of zinc, shPKA significantly suppressed ALP activity and mineralization (Fig. 3D, E), suggesting that PKA inhibition abolishes zinc-induced pro-osteogenic effects in hBMSCs.

\section{PKA inhibitor reverses zinc-mediated activation of PKA downstream genes during the osteogenic differentiation}

To confirm whether transcriptional activity and protein level of phospho-CREB are regulated by PKA signaling in the presence of zinc, we treated $\mathrm{ZnSO}_{4}$ and then inhibited PKA signaling using $\mathrm{H}-89$ during osteogenic differentiation for 5 days. As a result, zinc-induced RUNX2 expression and phosphorylation of CREB were suppressed by inhibition of PKA (Fig. 4A). As phosphorylation of CREB is known to lead to nuclear import [28,29], we investigated the cellular localization of phospho-CREB. We observed that PKA inhibition blocked the nuclear translocation of phosphoCREB induced by $\mathrm{ZnSO}_{4}$ during osteogenic differentiation (Fig. 4B, C). Next, we examined whether zinc-mediated cellular localization of CREB could regulate CREB transcription levels. A luciferase reporter assay showed that $\mathrm{ZnSO}_{4}$ increased transcription activity of CREB. In contrast, PKA inhibition significantly repressed its activity (Fig. 4D). Previously, $\mathrm{ZnSO}_{4}$ was also found to activate AKT and $\beta$ CATENIN during osteogenic differentiation of hBMSCs 

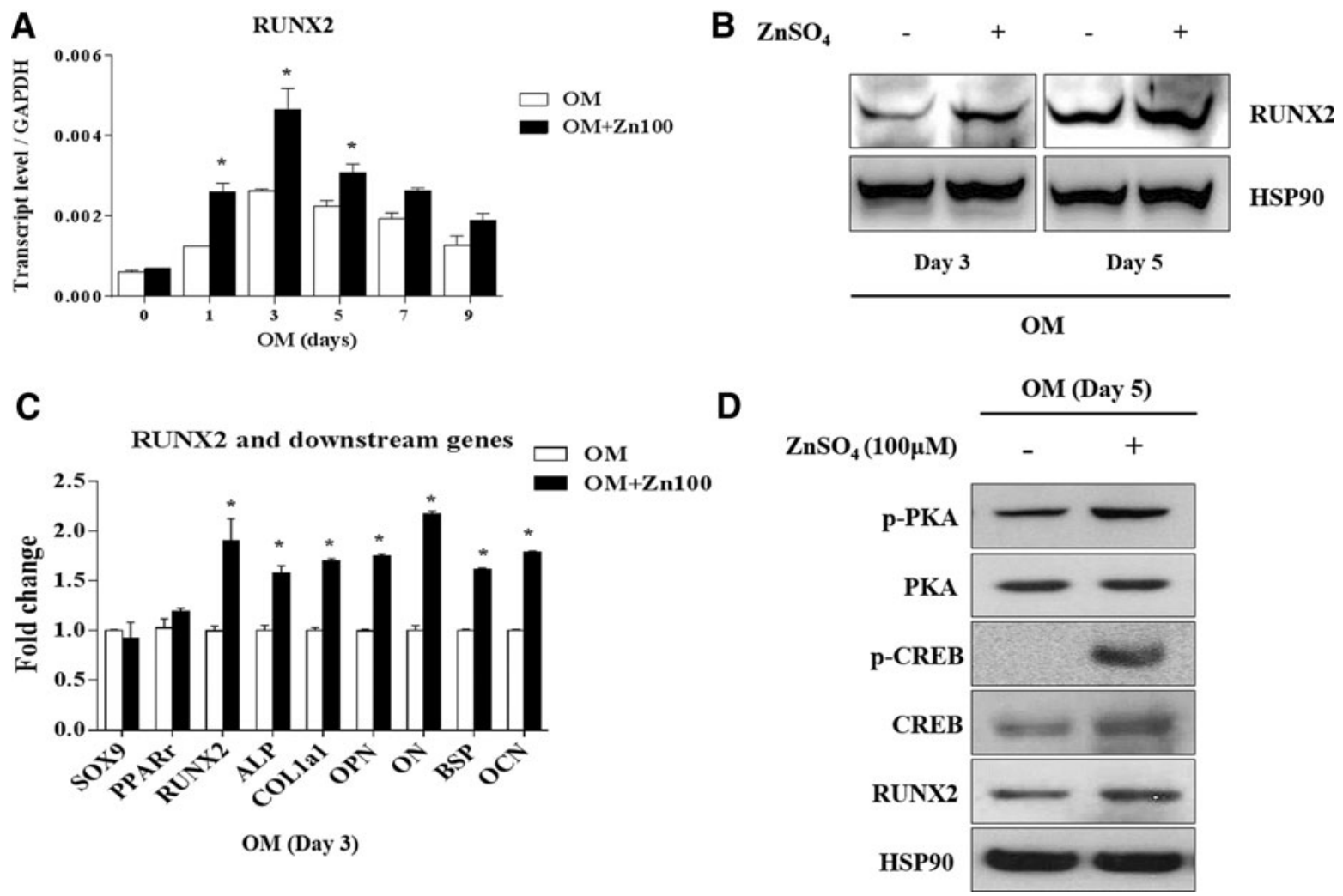

FIG. 2. Zinc increases expression of RUNX2 and its target genes through phosphorylation of PKA during osteogenic differentiation of hBMSCs. (A) hBMSCs were cultured in the presence or absence of $\mathrm{ZnSO}_{4}(100 \mu \mathrm{M})$ under osteogenic conditions. During osteogenesis, total RNA was extracted from the cultured hBMSCs, and mRNA levels for RUNX2 were measured by qPCR. (B) hBMSCs were incubated with OM and $\mathrm{ZnSO}_{4}(100 \mu \mathrm{M})$. After 3 and 5 days, total proteins were extracted from each group and evaluated by western blotting with anti-RUNX2 antibody. (C) On day 3 of osteogenesis, mRNA expression of osteoblast marker genes was assessed via qPCR. The results are expressed relative to each mRNA (D) Protein levels of PKA, phospho-CREB, and RUNX2 were quantified by western blot analysis. Data are presented as the mean \pm SD of at least three independent experiments. $* P<0.05$ compared with control. qPCR, quantitative real-time polymerase chain reaction.

(Supplementary Fig. S3B). It has also been reported that PKA can induce phosphorylation of AKT and activate $\beta$ CATENIN by phosphorylating GSK-3 $\beta$ [30,31]. PKA inhibition likewise suppressed phosphorylation of AKT and GSK-3 $\beta$ induced by $\mathrm{ZnSO}_{4}$ (Supplementary Fig. S5A). Moreover, while zinc increased the transcription activities of AKT and $\beta$-CATENIN, their activities were repressed by PKA inhibition (Supplementary Fig. S5B, C). These results are consistent with previous reports that PKA signaling activates AKT and $\beta$-CATENIN. Overall, these results suggested that zinc-induced osteogenesis is mediated by the PKA signaling pathway.

\section{Zinc activates PKA signaling pathway via increasing intracellular $c A M P$}

It has been well-established that the activity of PKA depends on cellular level of cAMP $[32,33]$. Therefore, we hypothesized that zinc regulates the PKA signaling pathway by increasing cellular cAMP levels during osteogenic differentiation in hBMSCs. To test this hypothesis, we measured the levels of intracellular cAMP activity in hBMSCs treated with $\mathrm{ZnSO}_{4}$. Zinc increased intracellular cAMP levels in a dose-dependent manner (Fig. 5A). However, the activities of PDEs, which is known to hydrolyze cAMP into inactive 5'-nucleotide monophosphates [34], showed no significant change during treatments with varying doses of $\mathrm{ZnSO}_{4}$ (Fig. 5B). These findings indicated that zinc activates the PKA signaling pathway by increasing intracellular cAMP levels with PDE-independent manner (Fig. 5C).

\section{Discussion}

In this series of experiments, we have demonstrated that zinc promotes phospho-CREB translocation into the nucleus by increasing PKA activity during osteogenesis in hBMSCs. This effect ultimately enhances expression of RUNX2 and activates osteoblast differentiation. Interestingly, in this study, zinc exposure was found to result in a dose-dependent increase in intracellular cAMP in OM, indicating that enhanced PKA activity may be caused by increased intracellular cAMP. Based on the results of this investigation, we propose that zinc promotes osteogenesis via its effects on the downstream signaling pathways of the cAMP-PKACREB axis.

Guo et al. have previously suggested that zinc deficiency may induce apoptosis in osteoblastic MC3T3-E1 cells and cause loss of bone mass [35]. However, there have been no 
A
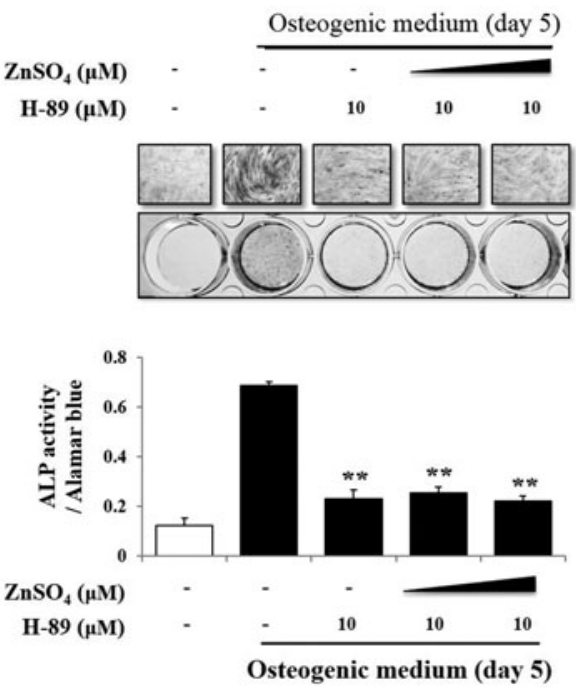

C

shCont. shPKA-1 shPKA-2

PKA
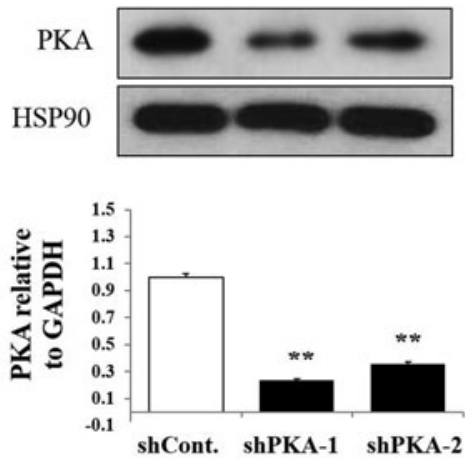

D
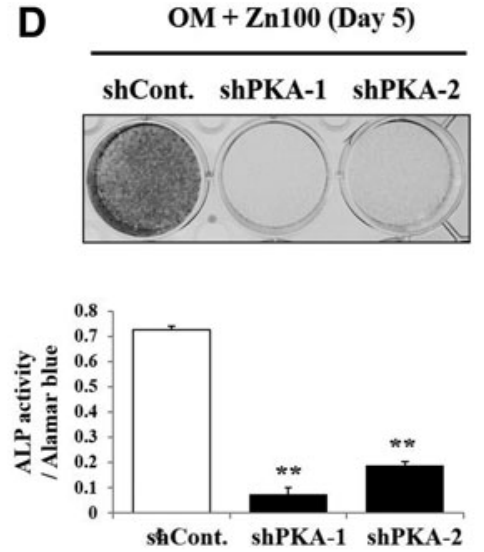

B
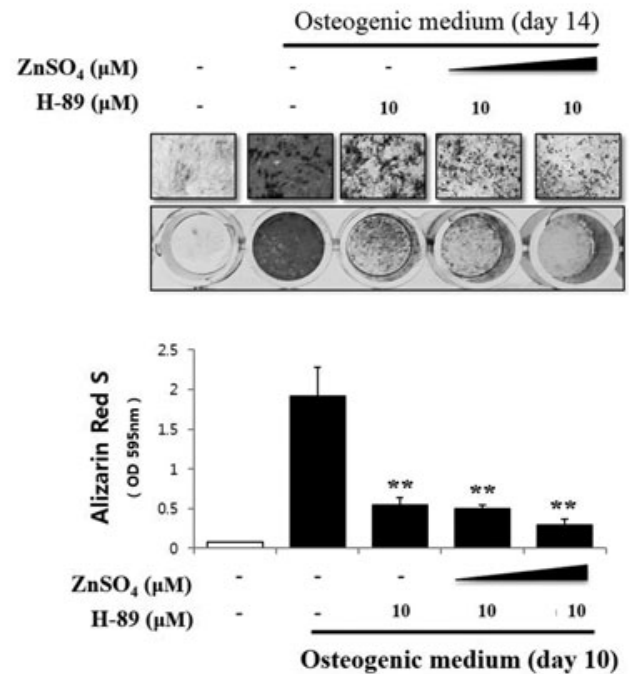

E $\mathrm{OM}+\mathrm{Zn} 100($ Day 14)

shCont. shPKA-1 shPKA-2
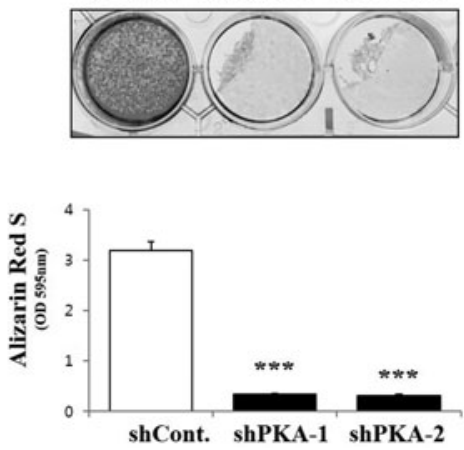

FIG. 3. Zinc exposure did not reverse suppression of osteogenesis by PKA inhibition in hBMSCs. hBMSCs $\left(4 \times 10^{4}\right.$ cells/ well in 24-well plates) were cultured with $\mathrm{OM}$, and $10-20 \mu \mathrm{M}$ of $\mathrm{ZnSO}_{4}$, followed by the addition of $\mathrm{H}-89(0.1 \mu \mathrm{M})$. $(\mathbf{A})$ ALP staining was performed (upper panel) and the amount of ALP activity was measured to determine the extent of early osteoblast differentiation (lower panel). ${ }^{*} * P<0.01$ compared with control treated with OM. (B) Alizarin red S staining was performed to detect mineral deposition (upper panel). For quantitative analysis of alizarin red $\mathrm{S}$ staining, absorbance was measured at $595 \mathrm{~nm}$ (lower panel). ${ }^{*} P<<0.01$ compared with control treated with OM. (C) The mRNA level of PKA was measured using qPCR (upper panel), and protein level was quantified with western blot analysis (lower panel) in lentiviral shCont- or shPKA-infected hBMSCs. $* * P<0.01$ compared with control treated with shCont. (D) At 5 days after induction of osteogenic differentiation with $\mathrm{ZnSO}_{4}(100 \mu \mathrm{M})$, ALP staining was performed (upper panel) and the amounts of ALP were measured in shCont- or shPKA-infected hBMSCs (lower panel). $* * P<0.01$ compared with control treated with shCont. (E) At 10 days, alizarin red S staining was performed for deposits of mineralization (upper panel). For quantitative analysis of alizarin red S staining, absorbance was measured at $595 \mathrm{~nm}$ (lower panel). Data are presented as the mean $\pm \mathrm{SD}$ of at least three independent experiments. $* * * P<0.001$ compared with control treated with shCont.

reports demonstrating effects of high-dose zinc on osteoblast apoptosis. In this investigation, we began by establishing a concentration of zinc $(100 \mu \mathrm{M})$ that was nontoxic to hBMSCs. Although a $100 \mu \mathrm{M}$ concentration of zinc was found to significantly activate ALP activity in hBMSCs, it was not observed to affect the viability of these cells (Fig. 1A, C). As shown in Figure 1B, the number of hBMSC colonies did not decrease following zinc treatment, indicating that the tested concentrations of zinc did not impact the capacity of hBMSCs for self-renewal. These findings likewise suggest that the osteogenic effects of zinc exposure do not result from enhanced self-renewal capacity.
Many previous studies have established that RUNX2 is a master regulator of osteoblast differentiation [36]. The functions of RUNX2 during osteoblast differentiation have been reported from both in vitro and in vivo studies. RUNX2 induces osteoblastic differentiation of MSCs by driving the expression of many bone matrix protein genes at the early stages of differentiation, while later RUNX2 inhibits osteoblast maturation [37]. Supporting the important role of RUNX2 in bone biology, RUNX2-deficient mice completely lack mature osteoblasts and mineralized bone formation [38]. Regulation of RUNX2 activity is therefore among the most important temporal events that occur during 
A

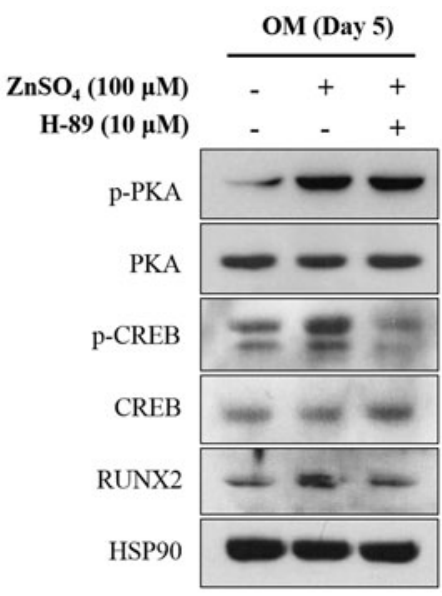

C

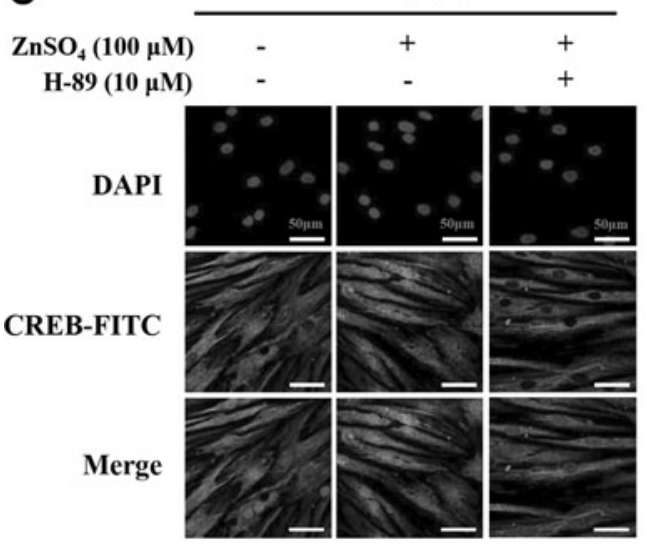

B
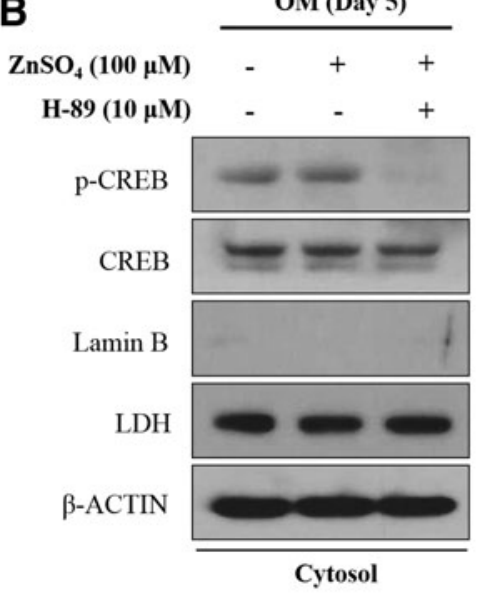

OM (Day 5)
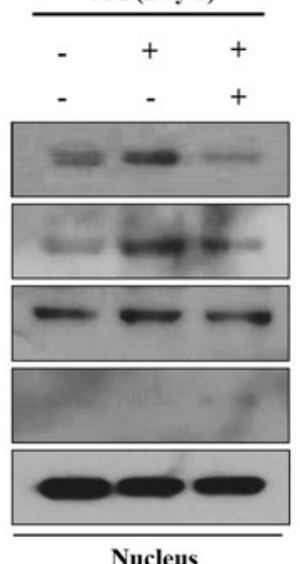

D
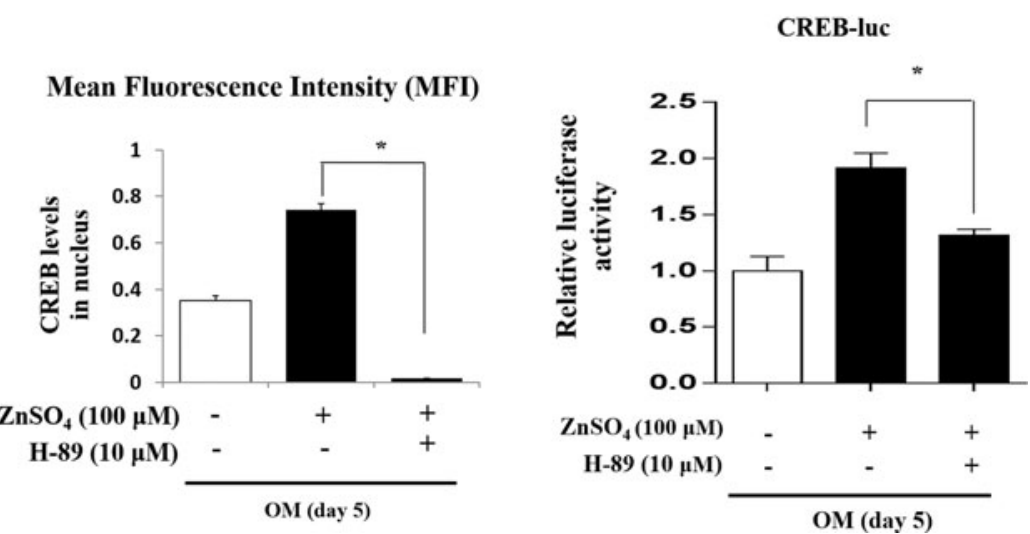

FIG. 4. PKA inhibition suppresses zinc-induced activation of PKA target genes during osteogenesis. hBMSCs were incubated with OM with $\mathrm{ZnSO}_{4}(100 \mu \mathrm{M})$ for 5 days. (A) Protein levels of PKA, phospho-CREB, phospho-AKT, active $\beta$ CATENIN, and RUNX2 were quantified by western blot analysis. (B) Cell lysates were prepared and then fractionated into nuclear and cytosolic extracts. Protein levels of phosphor-CREB and CREB were analyzed by western blot analysis. Lamin B protein level was used as a loading control for nuclear extracts, and the protein level of lactate dehydrogenase was used as a loading control for cytosolic extracts. (C) Immunocytochemistry was performed to observe the nuclear and cytosolic localization of CREB protein. The nucleus was stained with DAPI, and CREB was stained with FITCconjugated secondary antibody. Images were obtained using confocal microscopy. Scale bar $=100 \mu \mathrm{m}$. MFI was measured using ImageJ for triplicates. ${ }^{*} P<0.05$. (D) Luciferase reporter assay was performed to observe CREB transcriptional activity in hBMSCs treated with zinc or H-89 for 5 days after induction of osteogenesis. Dual-luciferase assays were performed with normalization to Renilla luminescence. Data are presented as the mean \pm SD of at least three independent experiments. $* P<0.05$ compared with control treated with $\mathrm{ZnSO}_{4}$. MFI, mean fluorescence intensity; FITC, fluorescein isothiocyanate; DAPI, 4,6-diamidino-phenyindole.

osteogenic differentiation of hMSCs. In mouse osteoblast cell lines, zinc supplementation increases RUNX2 expression at the mRNA and protein levels, while zinc deficiency reduces RUNX2 expression and nuclear RUNX2 protein levels during osteogenic differentiation [39]. Similarly, rats fed a zinc-free diet have been reported to have significantly decreased expression of RUNX2 mRNA measured from the distal femur [40]. Given the central role of RUNX2 in osteogenic differentiation and the evidence that zinc likewise may strongly influence osteogenesis, we hypothesized that zinc might contribute to regulation of RUNX2 during osteogenic differentiation of human mesenchymal stem cells, In this investigation, we aimed to further explore this hypothesis and clarify the molecular mechanisms responsible for the effects of zinc on osteogenic differentiation.
Consistent with previous reports, in this study, mRNA and protein levels of RUNX2 were found to increase upon zinc treatment as osteoblast differentiation progressed (Fig. 2A, B). These findings suggested that zinc accelerates osteoblast differentiation in human mesenchymal stem cells. However, as shown in Figure 4D, the transcriptional activity of CREB was not fully inhibited by PKA inhibitor during zinc treatment. Some residual activity was found to persist after treatment with PKA inhibitor. We propose that other signaling pathways, apart from PKA, may also partly contribute to CREB activation in response to zinc treatment, although these other pathway effects might not be sufficient to enhance osteoblast differentiation. Previous study reported that zinc deficiency leads to decreased osteoblastogenesis due to reduced Runx 2 expression through the 

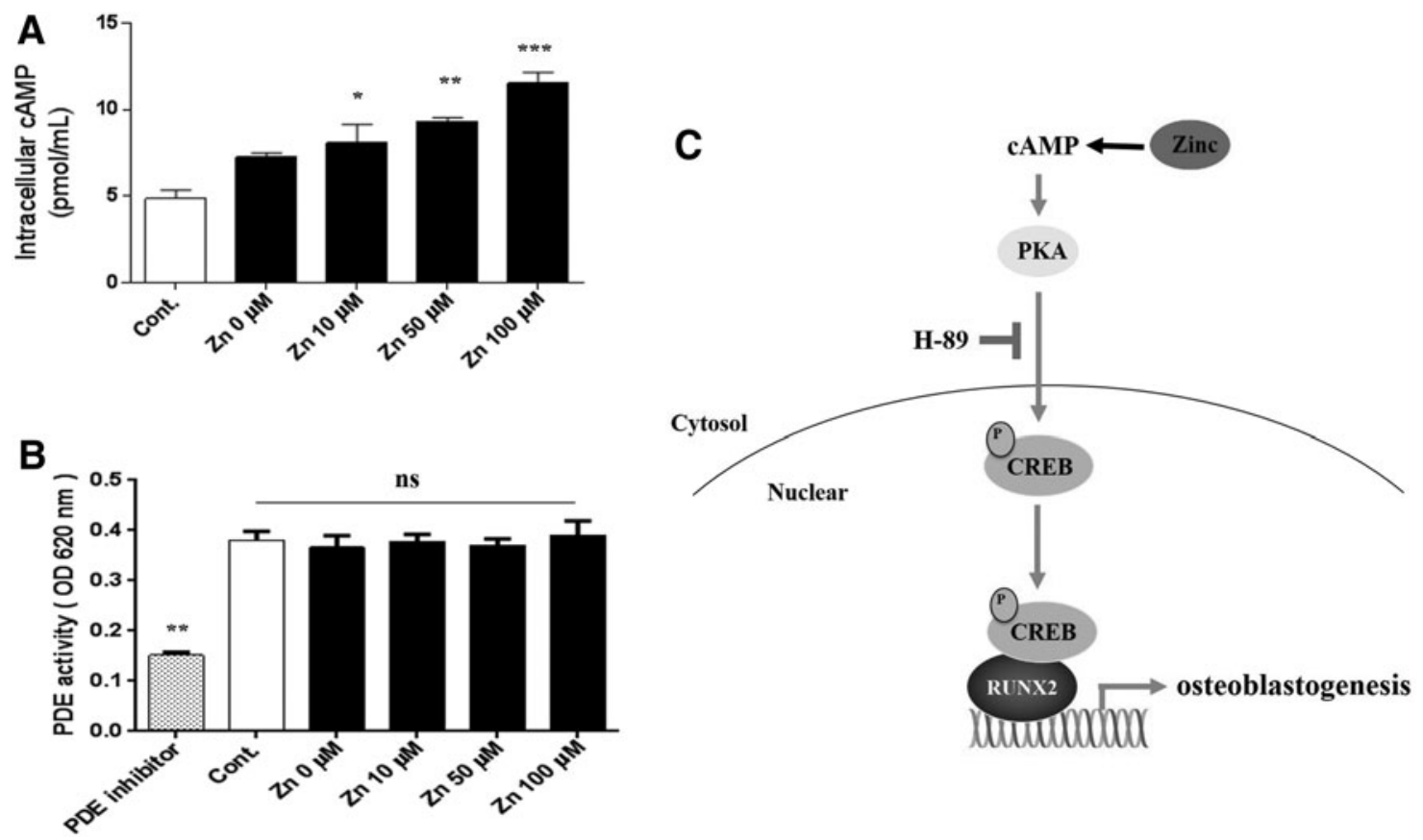

FIG. 5. Zinc increases intracellular cAMP in a dose-dependent manner during osteogenesis. (A) ELISA confirmed intracellular cAMP production from hBMSCs treated with zinc during osteogenesis for 15 min. Intracellular cAMP was significantly increased by zinc in dose-dependent manner $(n=3)$. $* P<0.05$; $* * P<0.01$; $* * * P<0.001$ compared with controls. (B) Zinc was treated in dose-dependent manner and PDE activity was measured as described above $(n=3$, in triplicate). Ten micrometers of PDE inhibitor (IBMX) was treated as a control. $* * P<0.01$ compared with control. (C) A proposed model for the mechanism by which ZnSO4 regulates osteogenic differentiation of hBMSCs through the PKACREB pathway. cAMP, cyclic adenosine monophosphate; PDE, phosphodiesterase.

inhibition of $\mathrm{Wnt} / \beta$-catenin signaling via the suppression of GSK $3 \beta$ inhibition and Akt activation preceded by the reduced level of SP-1 protein [40]. However, in this study, neither AKT inhibitor nor Wnt inhibitor (DKK1) completely reversed the osteogenic effects of zinc (Supplementary Fig. 4), although protein levels of $\mathrm{p}$-AKT and active $\beta$-catenin were increased during osteoblast differentiation (Supplementary Fig. S3B). In addition, the protein levels and transcriptional activities of p-AKT and $\beta$-catenin were partially suppressed by PKA inhibitor (Supplementary Fig. S5). These results indicated that zinc-induced AKT and $\beta$-catenin activities are mediated by the PKA signaling pathway, not by IGF or WNT signaling and PKA signaling may probably the main mechanism in the zinc effect.

Levels of intracellular cAMP are controlled by a balance between its synthesis by adenylate cyclase (AC) [41] and its hydrolysis into inactive ATP by PDE [34]. So far, controversy exists as to whether zinc influences intracellular cAMP level. It has been reported that zinc could prevent degradation of cAMP through inhibition of PDE activity in Sf9 cells [42], whereas zinc also inhibits forskolin-stimulated AC activity in a mouse neuroblastoma cell line [43]. However, our results demonstrate that zinc dose-dependently increases intracellular cAMP levels in hBMSC. We checked whether zinc could inhibit PDE activity in hBMSC, thus promoting intracellular cAMP level. However, zinc did not affect PDE activity in hBMSCs (Fig. 5A, B), suggesting that the increase of intracellular cAMP after zinc treatment was independent with $\mathrm{PDE}$ and may be related with AC activity.

Zinc is one of the nutrients essential for maintenance of bone health. In one animal model, zinc deficiency was re- ported to cause multiple bone abnormalities, including disturbances of bone formation, growth, and mineralization, thereby resulting in osteoporosis [44]. However, oral administration of zinc sulfate induced calcium contents in newborn rats [45]. In addition, when synthetic bone grafts incorporating zinc were implanted in the femoral condyles of ovariectomized rats, bone mass and bone mechanical properties were found to significantly improve in these animals [46]. Despite these previous studies, the mechanisms accounting for zinc-induced osteogenesis in hBMSCs had previously remained largely unclear. In this study, we present the results of a series of experiments which reveal the effects of zinc on regulation of the cAMP-PKA-CREB axis during osteoblast differentiation.

Osteoporosis is caused by an imbalance between osteoblast and osteoclast activity, which ultimately results in bone loss [47]. Among the drugs currently available for treating osteoporosis, most have only one effect domain, such as inhibition of bone resorption. A drug that only reduces resorption cannot restore bone loss previously caused by osteoclast activity. Therefore, a new strategy for enhanced treatment of osteoporosis is the development of drugs with dual effects, of not only blocking bone resorption but also promoting bone formation. Such drugs should also be cost-effective in order for these therapies to be available to all people who could benefit from using them. With respect to considerations of cost-effectiveness, zinc is notably inexpensive. In addition, several reports have indicated that zinc reduces osteoclast resorption activity [48]. In a previous study conducted by our group, we found that 
zinc inhibited osteoclast differentiation by suppression of the NFATc1 signaling pathway [49]. Subsequently, in this study, we explored the possibility that zinc also acts to effectively promote bone formation, and we established its molecular mechanism. Remarkably, zinc has now been shown both to increase bone mass and to decrease bone loss.

In conclusion, in this study, we report that zinc exerts osteogenic effects in hBMSCs by activation of RUNX2 via the cAMP-PKA-CREB signaling pathway. These findings provide mechanistic insights into the crucial role of zinc in bone formation. Zinc supplementation offers promise as a potential pharmaceutical therapy for osteoporosis and other bone loss conditions, particularly given the evidence from this study and others of its multimodal effects on bone resorption as well as bone formation.

\section{Acknowledgments}

This research was supported by Basic Science Research Program through the National Research Foundation of Korea (NRF) funded by the Ministry of Education (no. NRF-2016R1D1A1B03933182) and from the Bio \& Medical Technology Development Program of the National Research Foundation (NRF) funded by the Ministry of Science, ICT \& Future Planning (NRF-2017M3A9E8029722).

\section{Author Disclosure Statement}

No competing financial interests exist.

\section{References}

1. Raisz LG and E Seeman. (2001). Causes of age-related bone loss and bone fragility: an alternative view. J Bone Miner Res 16:1948-1952.

2. Rodan GA. (2003). The development and function of the skeleton and bone metastases. Cancer 97:726-732.

3. Roodman GD. (2004). Mechanisms of bone metastasis. N Engl J Med 350:1655-1664.

4. Huang W, S Yang, J Shao and YP Li. (2007). Signaling and transcriptional regulation in osteoblast commitment and differentiation. Front Biosci 12:3068-3092.

5. Grundberg E, H Brandstrom, KC Lam, S Gurd, B Ge, E Harmsen, A Kindmark, O Ljunggren, H Mallmin, O Nilsson and T Pastinen. (2008). Systematic assessment of the human osteoblast transcriptome in resting and induced primary cells. Physiol Genomics 33:301-311.

6. Hamidouche Z, O Fromigue, J Ringe, T Haupl, P Vaudin, JC Pages, S Srouji, E Livne and PJ Marie. (2009). Priming integrin alpha5 promotes human mesenchymal stromal cell osteoblast differentiation and osteogenesis. Proc Natl Acad Sci U S A 106:18587-18591.

7. Fong L, K Tan, C Tran, J Cool, MA Scherer, R Elovaris, $\mathrm{P}$ Coyle, BK Foster, AM Rofe and CJ Xian. (2009). Interaction of dietary zinc and intracellular binding protein metallothionein in postnatal bone growth. Bone 44:1151-1162.

8. Leek JC, JB Vogler, ME Gershwin, MS Golub, LS Hurley and AG Hendrickx. (1984). Studies of marginal zinc deprivation in rhesus monkeys. V. Fetal and infant skeletal effects. Am J Clin Nutr 40:1203-1212.

9. Prasad AS. (1983). Clinical, biochemical and nutritional spectrum of zinc deficiency in human subjects: an update. Nutr Rev 41:197-208.
10. Eberle J, S Schmidmayer, RG Erben, M Stangassinger and HP Roth. (1999). Skeletal effects of zinc deficiency in growing rats. J Trace Elem Med Biol 13:21-26.

11. Litchfield TM, Y Ishikawa, LN Wu, RE Wuthier and GR Sauer. (1998). Effect of metal ions on calcifying growth plate cartilage chondrocytes. Calcif Tissue Int 62:341-349.

12. Chen D, LC Waite and WM Pierce, Jr. (1999). In vitro effects of zinc on markers of bone formation. Biol Trace Elem Res 68:225-234.

13. Yamaguchi M and T Matsui. (1996). Stimulatory effect of zinc-chelating dipeptide on deoxyribonucleic acid synthesis in osteoblastic MC3T3-E1 cells. Peptides 17:1207-1211.

14. Strause L, P Saltman, KT Smith, M Bracker and MB Andon. (1994). Spinal bone loss in postmenopausal women supplemented with calcium and trace minerals. J Nutr 124: 1060-1064.

15. Freudenheim JL, NE Johnson and EL Smith. (1986). Relationships between usual nutrient intake and bone-mineral content of women 35-65 years of age: longitudinal and cross-sectional analysis. Am J Clin Nutr 44:863-876.

16. Fukada T, N Civic, T Furuichi, S Shimoda, K Mishima, H Higashiyama, Y Idaira, Y Asada, H Kitamura, et al. (2008). The zinc transporter SLC39A13/ZIP13 is required for connective tissue development; its involvement in BMP/ TGF-beta signaling pathways. PLoS One 3:e3642.

17. Hojyo S, T Fukada, S Shimoda, W Ohashi, BH Bin, $\mathrm{H}$ Koseki and T Hirano. (2011). The zinc transporter SLC39A14/ZIP14 controls G-protein coupled receptormediated signaling required for systemic growth. PLoS One 6:e18059.

18. Lee S, DS Yoon, S Paik, KM Lee, Y Jang and JW Lee. (2014). microRNA-495 inhibits chondrogenic differentiation in human mesenchymal stem cells by targeting Sox9. Stem Cells Dev 23:1798-1808.

19. Yoon DS, YH Kim, HS Jung, S Paik and JW Lee. (2011). Importance of Sox 2 in maintenance of cell proliferation and multipotency of mesenchymal stem cells in low-density culture. Cell Prolif 44:428-440.

20. Yoon DS, YH Kim, S Lee, KM Lee, KH Park, Y Jang and JW Lee. (2014). Interleukin-6 induces the lineage commitment of bone marrow-derived mesenchymal multipotent cells through down-regulation of Sox 2 by osteogenic transcription factors. FASEB J 28:3273-3286.

21. Yoon DS, Y Choi, Y Jang, M Lee, WJ Choi, SH Kim and JW Lee. (2014). SIRT1 directly regulates SOX2 to maintain self-renewal and multipotency in bone marrow-derived mesenchymal stem cells. Stem Cells 32:3219-3231.

22. Yoon DS, Y Choi, SM Choi, KH Park and JW Lee. (2015). Different effects of resveratrol on early and late passage mesenchymal stem cells through beta-catenin regulation. Biochem Biophys Res Commun 467:1026-1032.

23. Byun MR, AR Kim, JH Hwang, KM Kim, ES Hwang and JH Hong. (2014). FGF2 stimulates osteogenic differentiation through ERK induced TAZ expression. Bone 58: 72-80.

24. Chen G, C Deng and YP Li. (2012). TGF-beta and BMP signaling in osteoblast differentiation and bone formation. Int J Biol Sci 8:272-288.

25. James AW. (2013). Review of signaling pathways governing MSC osteogenic and adipogenic differentiation. Scientifica (Cairo) 2013:684736.

26. Miao D, XK Tong, GK Chan, D Panda, PS McPherson and D Goltzman. (2001). Parathyroid hormone-related peptide stimulates osteogenic cell proliferation through protein 
kinase $\mathrm{C}$ activation of the Ras/mitogen-activated protein kinase signaling pathway. J Biol Chem 276:32204-32213.

27. Wang S, J Mu, Z Fan, Y Yu, M Yan, G Lei, C Tang, Z Wang, Y Zheng, J Yu and G Zhang. (2012). Insulin-like growth factor 1 can promote the osteogenic differentiation and osteogenesis of stem cells from apical papilla. Stem Cell Res 8:346-356.

28. Jin XL and C O'Neill. (2010). The presence and activation of two essential transcription factors (cAMP response element-binding protein and cAMP-dependent transcription factor ATF1) in the two-cell mouse embryo. Biol Reprod 82:459-468.

29. Stevenson AS, L Cartin, TL Wellman, MH Dick, MT Nelson and KM Lounsbury. (2001). Membrane depolarization mediates phosphorylation and nuclear translocation of CREB in vascular smooth muscle cells. Exp Cell Res 263:118-130.

30. Bir SC, Y Xiong, CG Kevil and J Luo. (2012). Emerging role of PKA/eNOS pathway in therapeutic angiogenesis for ischaemic tissue diseases. Cardiovasc Res 95:7-18.

31. Zhang J, JR Shemezis, ER McQuinn, J Wang, M Sverdlov and A Chenn. (2013). AKT activation by N-cadherin regulates beta-catenin signaling and neuronal differentiation during cortical development. Neural Dev 8:7.

32. Delghandi MP, M Johannessen and U Moens. (2005). The cAMP signalling pathway activates CREB through PKA, p38 and MSK1 in NIH 3T3 cells. Cell Signal 17:1343-1351.

33. Skalhegg BS and K Tasken. (2000). Specificity in the cAMP/ PKA signaling pathway. Differential expression,regulation, and subcellular localization of subunits of PKA. Front Biosci 5:D678-D693.

34. von Bulow V, L Rink and H Haase. (2005). Zinc-mediated inhibition of cyclic nucleotide phosphodiesterase activity and expression suppresses TNF-alpha and IL-1 beta production in monocytes by elevation of guanosine $3^{\prime}, 5^{\prime}$-cyclic monophosphate. J Immunol 175:4697-4705.

35. Guo B, M Yang, D Liang, L Yang, J Cao and L Zhang. (2012). Cell apoptosis induced by zinc deficiency in osteoblastic MC3T3-E1 cells via a mitochondrial-mediated pathway. Mol Cell Biochem 361:209-216.

36. Komori T. (2006). Regulation of osteoblast differentiation by transcription factors. J Cell Biochem 99:1233-1239.

37. Liu W, S Toyosawa, T Furuichi, N Kanatani, C Yoshida, Y Liu, M Himeno, S Narai, A Yamaguchi and T Komori. (2001). Overexpression of Cbfa1 in osteoblasts inhibits osteoblast maturation and causes osteopenia with multiple fractures. J Cell Biol 155:157-166.

38. Adhami MD, H Rashid, H Chen, JC Clarke, Y Yang and A Javed. (2015). Loss of Runx2 in committed osteoblasts impairs postnatal skeletogenesis. J Bone Miner Res 30:7182.

39. Kwun IS, YE Cho, RA Lomeda, HI Shin, JY Choi, YH Kang and JH Beattie. (2010). Zinc deficiency suppresses matrix mineralization and retards osteogenesis transiently with catch-up possibly through Runx 2 modulation. Bone 46:732-741.

40. Hie M, N Iitsuka, T Otsuka, A Nakanishi and I Tsukamoto. (2011). Zinc deficiency decreases osteoblasts and osteoclasts associated with the reduced expression of Runx 2 and RANK. Bone 49:1152-1159.

41. Wellinghausen N, C Driessen and L Rink. (1996). Stimulation of human peripheral blood mononuclear cells by zinc and related cations. Cytokine 8:767-771.

42. Percival MD, B Yeh and JP Falgueyret. (1997). Zinc dependent activation of cAMP-specific phosphodiesterase (PDE4A). Biochem Biophys Res Commun 241:175-180.

43. Klein C, RK Sunahara, TY Hudson, T Heyduk and AC Howlett. (2002). Zinc inhibition of cAMP signaling. J Biol Chem 277:11859-11865.

44. Hyun TH, E Barrett-Connor and DB Milne. (2004). Zinc intakes and plasma concentrations in men with osteoporosis: the Rancho Bernardo Study. Am J Clin Nutr 80:715721.

45. Ma ZJ and M Yamaguchi. (2000). Alternation in bone components with increasing age of newborn rats: role of zinc in bone growth. J Bone Miner Metab 18:264-270.

46. Samira J, M Saoudi, K Abdelmajid, O Hassane, R Treq, E Hafed, E Abdelfatteh and K Hassib. (2015). Accelerated bone ingrowth by local delivery of Zinc from bioactive glass: oxidative stress status, mechanical property, and microarchitectural characterization in an ovariectomized rat model. Libyan J Med 10:28572.

47. Tanaka Y, S Nakayamada and Y Okada. (2005). Osteoblasts and osteoclasts in bone remodeling and inflammation. Curr Drug Targets Inflamm Allergy 4:325-328.

48. Moonga BS and DW Dempster. (1995). Zinc is a potent inhibitor of osteoclastic bone resorption in vitro. J Bone Miner Res 10:453-457.

49. Park KH, B Park, DS Yoon, SH Kwon, DM Shin, JW Lee, HG Lee, JH Shim, JH Park and JM Lee. (2013). Zinc inhibits osteoclast differentiation by suppression of $\mathrm{Ca} 2+-$ Calcineurin-NFATc1 signaling pathway. Cell Commun Signal 11:74.

Address correspondence to: Jin Woo Lee, MD, PhD Department of Orthopaedic Surgery Yonsei University College of Medicine 250, Seongsanno, Seodaemun-gu Seoul, 120-752 South Korea

E-mail: ljwos@yuhs.ac

Received for publication February 7, 2018

Accepted after revision May 30, 2018

Prepublished on Liebert Instant Online May 31, 2018 